\title{
Simulação dos Impactos das Mudanças Climáticas na Vazão da Bacia do Ribeirão do Feijão - SP
}

\section{Impact of climate change on Feijão River basin flow, São Paulo State, Brazil}

\author{
Paulo Ponce Arroio Junior ${ }^{1}$ e Frederico Fabio Mauad ${ }^{2}$ \\ ${ }^{1}$ Programa de Pós-Graduação em Ciências da Engenharia Ambiental, Escola de Engenharia de São Carlos, Universidade de São Paulo, \\ São Carlos, SP. Brasil \\ pauloarroio@sc.usp.br \\ ${ }^{2}$ Departamento de Hidráulica e Saneamento, Escola de Engenharia de São Carlos, Universidade de São Paulo. São Carlos, SP. Brasil \\ mauadffm@sc.usp.br
}

\section{RESUMO}

As mudanças climáticas ocasionadas pelo aumento da concentração de GEEs na atmosfera poderão acarretar impactos significativos na dinâmica das bacias hidrográficas nas próximas décadas. Nesse sentido, o modelo SWAT foi aplicado para avaliar os impactos destas mudanças na vazãa da bacia do Ribeirão do Feijão, localizada no interior do Estado de São Paulo. Utilizou-se o gerador climático estocástico LARS-WG para gerar séries sintéticas diárias sob os cenários B1, A1B e A2 do IPCC. Tais séries foram inseridas no modelo SWAT calibrado com o objetivo de avaliar o comportamento da bacia nos períodos de 2050-2059 e 2090-2099. Para a vazão mensal, as etapas de calibração e validação do SWAT geraram coeficientes de Nash-Sutcliffe de 0,73 e 0,78, respectivamente, enquanto para a vąão diária os coeficientes foram de 0,57 e 0,63. Nas simulações para a década de 2050 foram observados aumentos na vaz̧ão em todos os cenários, com exceção do cenário A2 no período seco, onde observou-se uma redução de -1,5\% a -3\% durante estes meses. Para a década de 2090 o cenário A1B apresentou redução de vaz̧ão de março a outubro, enquanto no cenário A2 tal redução foi observada entre fevereiro e outubro, chegando a um decréscimo de -10\% quando comparado às vaz̃ões atuais. No geral, os resultados indicaram que a bacia do Ribeirão do Feijão é bastante vulnerável às potenciais alterações climáticas, estando sujeita a possiveis problemas de disponibilidade bídrica no futuro.

Palavras Chave: SWAT. Hidrologia. Mudanças climáticas. Modelagem

\begin{abstract}
Climate changes brought on by increasing greenhouse gases in the atmosphere are expected to have a significant effect on small watersheds during the 21st century. SWAT has been used with the aim of evaluating the impact of climate change on the flow of the Feijäo River basin, in São Paulo State, Brazil. Stochastic weather generator LARS-WG was used to simulate time-series of daily weather under climate scenarios B1, A1B and A2 of IPCC. These data were used at calibrated SWAT model to simulate basin behavior over the period 2050-2059 and 2090-2099. Model calibration and validation resulted in Nash-Sutcliffe coefficients of 0.73 and 0.78, respectively, for monthly flow and 0.57 and 0.63, for daily flow. Simulations for 2050 'sshowed increases of flow for all scenarios, except A2 during dry season (reduction of -1.5\% to -3\%). For 2090's, A1B scenario showed reduction from march to october, while in A2 scenario reduction was between february and october, reaching a decrease of $10 \%$ compared to current flow. The results indicate that the basin is very sensitive to potential climate changes, being subject to possible problems of water availability in the future.
\end{abstract}

Keywords: SWAT. Hydrology. Climate change modeling 


\section{INTRODUÇÃO}

O contínuo desenvolvimento da sociedade e a intensa utilização de combustíveis fósseis ocorrida após a Revolução Industrial ocasionaram um aumento considerável dos gases de efeito estufa (GEEs) na atmosfera do planeta. Com base nos dados do Painel Intergovernamental sobre Mudanças Climáticas (IPCC), desde o ano de 1750 constata-se um aumento contínuo das concentrações atmosféricas de gases como dióxido de carbono (CO2) e metano (CH4), sendo tal aumento atribuído às atividades humanas. No ano de 2011 as concentrações destes GEEs foram de 391 ppm e 1803 ppb, respectivamente, o que representa um acréscimo de $40 \%$ e $150 \%$ em relação a era pré -industrial (IPCC, 2013).

Vários estudos apontam que este aumento de GEEs da atmosfera é o principal responsável pelas alterações no equilíbrio energético do sistema climático, atribuindo ao acúmulo destes gases um aquecimento atmosférico adicional que resulta no aumento da temperatura média global. Pesquisadores do IPCC estimam que a temperatura média do planeta aumentou em $0,85^{\circ}$ Centre os anos de 1880 e 2012 , e projetam para os próximos anos um aquecimento de $0,12^{\circ} \mathrm{C}$ por década. Além disso, tais mudanças acarretariam verões mais secos e quentes e significativas alterações nos volumes e distribuição das chuvas, concentrando as precipitações em algumas regiões e tornando-as escassas em outras (IPCC, 2013).

Segundo Marengo (2008), as principais ferramentas utilizadas pelos centros de pesquisa do clima para projetar cenários de fenômenos meteorológicos futuros são os modelos de circulação geral (GCMs). Os GCMs são representações dos processos físicos na atmosfera, no oceano e na superfície da terra, que simulam a resposta do clima global terrestre ao aumento nas concentrações de GEEs. Esses modelos descrevem a atmosfera utilizando um grid tridimensional sobre o planeta, tendo resolução global variando entre 250 e 600 km (MELLO et al., 2008).

Nesse contexto, é crescente a preocupação de órgãos gestores e círculos científicos com relação aos impactos destas mudanças sobre os recursos hídricos. Com o aumento da temperatura espera-se que as alterações no ciclo hidrológico tornem-se cada vez mais acentuadas. Os montantes pluviométricos e sua distribuição temporal e espacial, bem como o processo de evapotranspiração, serão diretamente influenciados, ocasionando mudanças nas taxas de infiltração e de geração de escoamento superficial. Estas alterações irão influenciar o armazenamento de água no solo e, consequentemente, a recarga dos aquíferos, afetando a disponibilidade de água, modificando o regime hídrico e alterando a resposta hidrológica das bacias hidrográficas (MARENGO, 2008; TUCCI, 2007). Tais impactos acarretariam implicações consideráveis nos sistemas operacionais, no planejamento e no gerenciamento das bacias hidrográficas, principalmente em países como o Brasil, onde a disponibilidade de água é bastante influenciada pelo clima.

Visando avaliar os impactos da mudança do clima no ciclo hidrológico, diversos estudos são realizados acoplando-se modelos de circulação geral (GCMs) da atmosfera a modelos hidrológicos. Entretanto, parte destes estudos tem se concen- trado nas escalas continental e global, sendo necessárias maiores investigações sobre como as possíveis mudanças nos padrões de temperatura e precipitação trarão implicações para bacias hidrográficas de pequeno e médio porte.

Nesse sentido, para avaliar os impactos dessas mudanças sobre os processos hidrológicos na escala de bacia, torna-se necessário o uso de modelos que operem a partir de uma concepção de base física dos processos envolvidos. Dentre estes modelos, o Soil and Water Assessment Tool (SWAT), desenvolvido pelo Agricultural Research Service e pela Texas A\&M University, destaca-se por incluir em suas rotinas de simulação componentes hidrológicos, climáticos, pedológicos e de uso da terra, permitindo avaliar os impactos de diferentes cenários na qualidade e quantidade dos recursos hídricos. O SWAT possui rotinas que permitem descrever como as alterações na temperatura e na precipitação afetam os processos de evapotranspiração, de geração de escoamento, de recarga subterrânea e outros elementos hidrológicos, configurando-se, portanto, como uma ferramenta de grande potencial para investigação de impactos decorrentes da mudança do clima.

Ademais, outra dificuldade inerente aos estudos de impactos da mudança do clima em bacias hidrográficas refere-se à baixa resolução espacial dos GCMs, os quais não conseguem representar adequadamente os aspectos climáticos locais e o microclima de pequenas áreas. Visando contornar este problema, foram criadas diversas técnicas matemáticas voltadas ao refinamento das projeções globais visando sua aplicação em escalas maiores, sendo tais procedimentos denominados downscaling ou redução de escala.

O refinamento das projeções climáticas provindas de GCMs através de downscaling possibilitou a obtenção de variáveis meteorológicas adequadas para estudos do impacto das mudanças climáticas, permitindo-se sanar o problema da discrepância de escalas em que operam os GCMs e os modelos hidrológicos. Nesse sentido, um grande número de pesquisas tem utilizado o SWAT para simulação de cenários futuros de mudança do clima, permitindo-se avaliar pontualmente o impacto destas mudanças em bacias hidrográficas. Em tais estudos, geralmente utilizam-se cenários climáticos gerados por modelos de circulação regional (RCMs), os quais por sua vez são derivados dos GCMs por meio de transformações de redução de escala denominadas downscaling dinâmico.

Ficklin et al. (2009) avaliaram a sensibilidade de uma bacia rural localizada na Califórnia às mudanças climáticas. Os autores construíram cenários de mudança do clima para o SWAT com base nas faixas de variação estimadas pelo IPCC para a concentração de CO2 (550 a 970 ppm), temperatura (+1,1 a + $\left.6.4^{\circ} \mathrm{C}\right)$ e precipitação $(0 \%, \pm 10 \%$, e $\pm 20 \%)$ nas próximas décadas, de modo a realizar uma simulação de vazão para daqui 50 anos. Wagesho et al. (2013) simularam o impacto das mudanças climáticas na vazão de duas bacias agrícolas na Etiópia. Para simulação de cenários, os autores utilizaram dados climáticos provenientes do Projeto CMIP3, o qual reúne informações de diversos modelos de circulação geral. Após a realização dos procedimentos de downscaling necessários para o refinamento dos dados visando sua aplicação em escala de bacia, realizaramse simulações para os cenários de altas emissões (A2) e médias 
emissões (A1B) de GEEs elaborados pelo IPCC. No trabalho de Zahabiyoun et al. (2013), as simulações foram realizadas na bacia do Rio Gharesou, no Irã, para o período de 2040-2069. Os autores construíram cenários de mudança do clima realizando-se downscaling dinâmico a partir dos dados do modelo de circulação HadCM3, tendo como base o cenário de altas emissões (A2) do IPCC.

No Brasil, são poucos os trabalhos utilizando o SWAT no contexto de mudanças climáticas. Lelis et al. (2011) aplicou o modelo na bacia do ribeirão São Bartolomeu (MG), efetuando simulações tendo como base o cenário A1B. Perazzoli et al. (2013) aplicou o SWAT na Bacia do Rio Concórdia (SC), visando simular os impactos na vazão e na produção de sedimentos. Os autores utilizaram os dados do GCM HadRM3P para representar o clima futuro com base nos cenários A2 (altas emissões) e B2 (baixas emissões) do IPCC, realizando simulações para 2071-2100. Valerio (2014) realizou simulações na bacia do rio Paraguaçu (BA) a partir do modelo calibrado para vazão diária. $\mathrm{O}$ autor também utilizou os cenários A2 e B2 do IPCC e realizou simulações utilizando projeções de diversos GCMs.

Tendo em vista a carência de estudos aplicando o SWAT para simulação de impactos da mudança do clima no país, o presente trabalho visa simular a influência destas mudanças na vazão da bacia do Ribeirão do Feijão, localizada no interior do Estado de São Paulo. Para geração dos cenários climáticos optou-se por utilizar um método de downscaling estatístico, no qual relacionam-se variáveis climáticas locais e variáveis em larga escala resultantes de GCMs por meio de funções estocásticas estimadas empiricamente a partir de observações históricas. Desse modo, diferentemente dos usuais métodos de downscaling dinâmico, onde os cenários são derivados de RCMs elaborados por centros de pesquisa do clima, o downscaling estatístico requer uma série climática de longa data adquirida no próprio local de estudo, a partir da qual são realizadas as transformações tendo como base os GCMs.
Espera-se que a aplicação desta metodologia possa contribuir para o entendimento dos impactos das mudanças climáticas em nível regional e local, fornecendo informações que possam subsidiar o planejamento dos recursos hídricos e o gerenciamento não somente da bacia em estudo, mas também de outras bacias cujas condições sejam semelhantes.

\section{MATERIAIS E MÉTODOS}

\section{Área de estudo}

A bacia do Ribeirão do Feijão está localizada no interior do Estado de São Paulo e pertence à Unidade de Gerenciamento de Recursos Hídricos 13 - Tietê/Jacaré, situando-se no alto curso do Rio Jacaré-Guaçu, conforme exposto na Figura 1. Com aproximadamente $241 \mathrm{~km}^{2}$ de extensão, tal bacia abrange parte dos municípios de São Carlos, Analândia e Itirapina, e tem como curso d'água principal o Ribeirão do Feijão, o qual possui aproximadamente $23,7 \mathrm{~km}$ de comprimento desde sua nascente principal até seu deságue no Rio Jacaré-Guaçu. O Ribeirão do Feijão é utilizado como manancial de captação desde a década de 70, tendo grande importância no abastecimento de água de São Carlos, além de ter importância regional por estar situado em área considerada como de recarga direta do Aquífero Guarani.

A bacia apresenta uma elevação média de $814 \mathrm{~m}$, onde a amplitude altimétrica é de 375 m (variação entre 650 $\mathrm{m}$ e $1025 \mathrm{~m}$ ). O uso da terra é voltado para atividade agrícola e pecuária, havendo também áreas de ocorrência de silvicultura. As áreas de pastagem correspondem a 29,7\% da área da bacia, seguidas pelas áreas florestadas $(26,1 \%)$, plantio de cana de açúcar $(17,4 \%)$, plantações de eucalipto $(13,6 \%)$, culturas agrícolas diversas $(10 \%)$, área urbanizada $(3,1 \%)$ dentre outros usos de menor ocorrência. Segundo Oliveira e Prado (1984), os Neossolos Quartzarênicos são os solos predominantes na bacia,

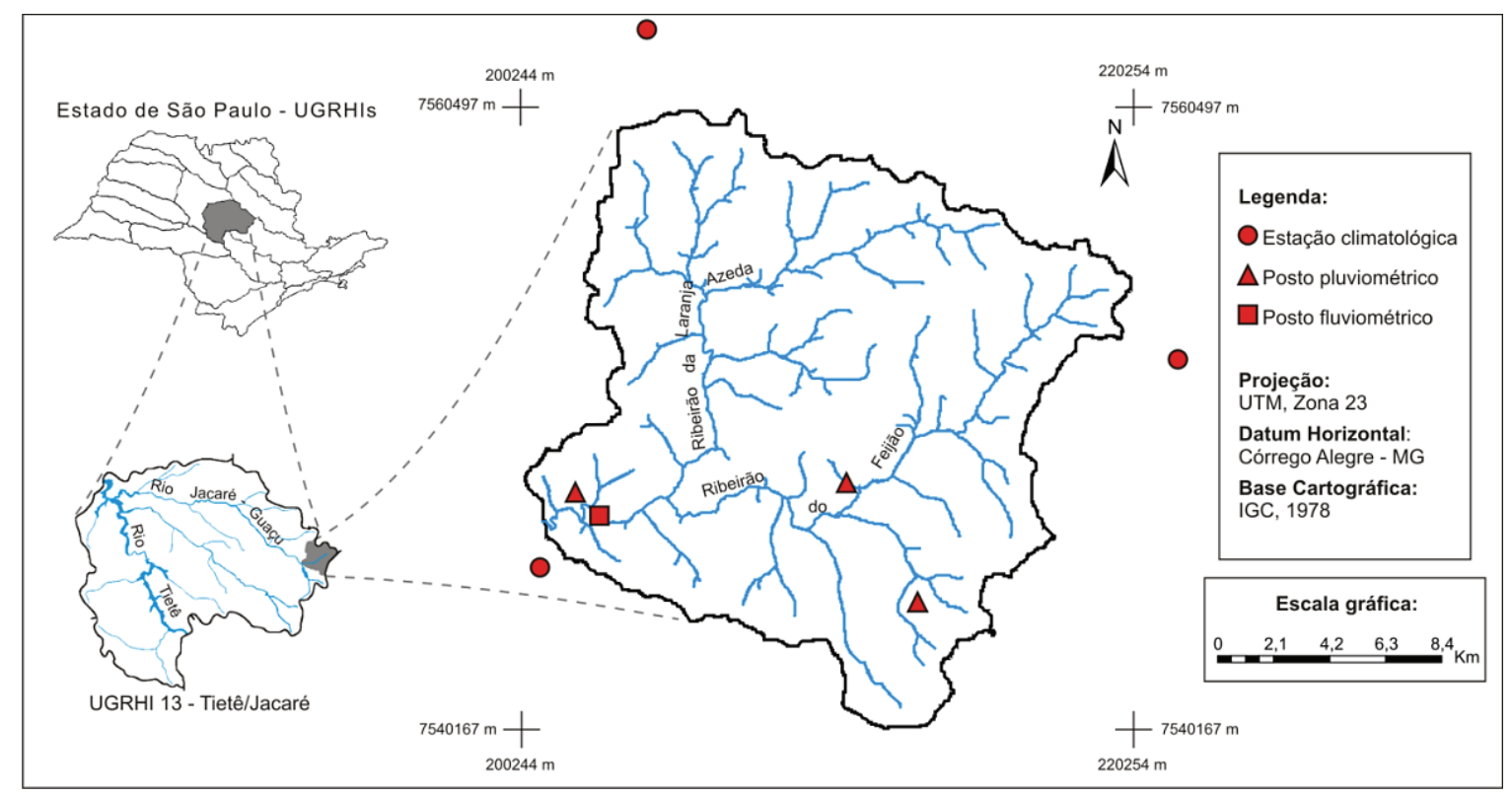

Figura 1 - Localização geográfica da Bacia do Ribeirão do Feijão e suas estações de monitoramento 
ocorrendo em mais de 40\% da área, havendo também presença significativa de Latossolos Vermelho-Amarelos (36\%) e, em menor ocorrência, manchas isoladas de Latossolos Vermelhos $(10,9 \%)$ e Neossolos Litólicos (6,1\%).

O clima da área de estudo é, segundo a classificação climática de Köppen, do tipo Cwa, ou seja, mesotérmico com inverno seco. A precipitação média anual é de $1.515 \mathrm{~mm}$, com mais de $78 \%$ das chuvas ocorrendo na estação chuvosa (outubro a março) e os $22 \%$ restantes no período seco (abril a setembro). Em relação à temperatura, a média anual é de $21,6^{\circ} \mathrm{C}$, onde o mês mais quente é fevereiro, com média mensal de $24,0^{\circ} \mathrm{C}$, e o mês mais frio é junho, com $17,6^{\circ} \mathrm{C}$ (ARROIO JUNIOR, 2013).

\section{Breve descrição do modelo SWAT}

O modelo SWAT opera em passo de tempo contínuo e apresenta parâmetros distribuídos, possibilitando que um grande número de diferentes processos físicos possa ser simulado em escala de bacia hidrográfica (SRINIVASAN; ARNOLD, 1994). Desse modo, o modelo é capaz de retratar os processos sequencialmente, num intervalo de tempo delimitado pelo usuário, fornecendo séries temporais como dado de saída (TIM, 1996). É possível a simulação de longos períodos de tempo, possibilitando computar os efeitos cumulativos de alterações na bacia, sejam estas variações de manejo, mudanças no uso e ocupação da terra, alterações climáticas, dentre outras.

O SWAT realiza no processo de modelagem a divisão da bacia em subunidades denominadas Unidades de Resposta Hidrológica (Hydrologic Response Units -HRU), que representam uma combinação única de tipo de solo, uso da terra e declividade. Tal subdivisão possibilita ao modelo refletir diferenças na evapotranspiração e outras condições hidrológicas para diferentes usos e solos. O escoamento é calculado em cada HRU e propagado para obter o escoamento para a bacia. Tal característica aumenta a precisão das predições e fornece uma melhor descrição física do balanço de água (ARNOLD et al., 2009).

Os fenômenos simulados pelo SWAT têm como principal fator controlador a equação geral do balanço hídrico, apresentada na equação 1 .

$$
\mathrm{SW}_{t}=\mathrm{SW}_{0}+\sum_{i=1}^{t}\left(\mathrm{R}_{\text {day }}-\mathrm{Q}_{\text {surf }}-\mathrm{E}_{\mathrm{a}}-\mathrm{w}_{\text {seep }}-\mathrm{Q}_{\mathrm{gw}}\right)
$$

Onde $\mathrm{SW}_{\mathrm{t}}$ é o conteúdo final de água no solo ( $\left.\mathrm{mm}\right), \mathrm{SW}_{0}$ é o conteúdo inicial de água no solo no dia i (mm), t é o tempo (dias), $R_{\text {day }}$ é a precipitação no dia $\mathrm{i}(\mathrm{mm}), \mathrm{Q}_{\text {surf }}$ é o escoamento superficial no dia i (mm), Ea é a evapotranspiração no dia i $(\mathrm{mm}), \mathrm{w}_{\text {seep }}$ é a quantidade de água que percola na zona vadosa $(\mathrm{mm})$ e $\mathrm{Q}_{\mathrm{gw}}$ é a quantidade de água que retorna ao curso d'água no dia i por escoamento subsuperficial $(\mathrm{mm})$.

Segundo Arnold et al. (2009), o sistema hidrológico simulado pelo SWAT compreende quatro principais componentes: os reservatórios superficiais, os reservatórios subsuperficiais, os reservatórios subterrâneos (aquíferos rasos ou livres) e os reservatórios subterrâneos (aquífero profundo). A contribuição desses reservatórios para o escoamento no canal provém do escoamento lateral a partir do perfil de solo e do escoamento de retorno do aquífero raso. O volume que percola do reservatório subsuperficial através do perfil de solo representa a recarga do aquífero raso, enquanto a água que percola para o aquífero profundo não retorna para o sistema.

A determinação do escoamento superficial no SWAT pode ser realizada através de dois métodos distintos, sendo eles o Método da Curva Número, elaborado pelo Soil Conservation Service (SCS), utilizado na configuração padrão do modelo, e o Método de Infiltração de Green e Ampt. O cálculo da evapotranspiração potencial pode ser realizado através de três métodos: Penman-Monteith (configuração padrão, mantida neste trabalho), Priestley-Taylor e Hargreaves. Segundo Arnold et al. (2009), a componente percolação do SWAT é calculada utilizando-se uma técnica de propagação do armazenamento combinada ao modelo do fluxo em fendas no solo para simular o escoamento através de cada camada do perfil. No cálculo do fluxo lateral, o SWAT utiliza um modelo de armazenamento cinemático baseado na equação de continuidade de massa, no qual considera o balanço hídrico com todo o segmento da encosta utilizado como volume de controle, em um fluxo bi-dimensional.

Ainda segundo Arnold et al. (2009), a vazão de pico é a vazão máxima que ocorre no canal devido a um dado evento de chuva. O SWAT calcula a vazão de pico através do Método Racional Modificado, fundamentado na suposição de que, se uma chuva de intensidade i inicia instantaneamente e continua indefinidamente, a vazão de pico do escoamento vai aumentar até o tempo de concentração, quando toda a sub-bacia estará contribuindo para o fluxo na saída. A equação do método racional é dada pela equação 2:

$$
\mathrm{q}_{\text {peak }}=\frac{\alpha_{\mathrm{tc}} \cdot \mathrm{Q}_{\text {surf }} \cdot \mathrm{A}}{3,6 \cdot \mathrm{t}_{\text {conc }}}
$$

Onde $\mathrm{q}_{\text {peak }}$ é a vazão de pico $\left(\mathrm{m} 3 . \mathrm{s}^{-1}\right), \alpha_{\mathrm{tc}}$ é a fração da precipitação diária que ocorre durante o tempo de concentração, $\mathrm{Q}_{\text {surf }}$ é o volume de escoamento superficial $\left(\mathrm{mm} \cdot \mathrm{ha}^{-1}\right)$, A é a área da bacia $\left(\mathrm{km}^{2}\right)$ e $t_{\text {conc }}$ é o tempo de concentração da bacia (h). O tempo de concentração é obtido pela soma do tempo do fluxo superficial (tempo necessário para que a água precipitada no ponto mais distante da sub-bacia alcance o canal) e do tempo do fluxo no canal (tempo necessário para que a água do canal alcance o exutório).

\section{Aplicação, calibração e validação do modelo SWAT para a bacia do Ribeirão do Feijão}

Para as operações de modelagem utilizou-se a versão 2009.93.7b do SWAT, a qual permite integração com o software ArcGIS 9.3. Visando representar toda a heterogeneidade existente nos sistemas modelados, o SWAT trabalha com uma grande quantidade de dados espaciais e não espaciais referentes à topografia, aos tipos de solo, ao uso da terra, ao clima, dentre outros.

A etapa inicial de simulação consiste na delimitação da bacia de drenagem e na sua divisão em sub-bacias. Para tal, o simulador requer como dado de entrada o Modelo Digital de Elevação (DEM), o qual foi gerado a partir da interpolação de 
curvas de nível de equidistância de 5 metros oriundas do mapeamento planialtimétrico realizado pelo Instituto Geográfico e Cartográfico do Estado de São Paulo (IGC),escala 1:10.000.

Em relação aos dados referentes aos solos, são necessários tanto dados espaciais quanto dados tabulares. Para a caracterização espacial foi utilizada a carta de solos da Quadrícula de São Carlos, folha SF 23-Y-A-I, referente ao levantamento pedológico semi-detalhado do Estado de São Paulo, publicado pela EMBRAPA, na escala de 1:100.000. Os dados tabulares: número de camadas, profundidade máxima, porosidade, densidade aparente, teor de carbono orgânico, granulometria, dentre outros, foram extraídos do memorial descritivo referente ao mapa de solos utilizado (OLIVEIRA; PRADO, 1984).

Com relação ao uso e ocupação da terra, são também necessários dados espaciais e tabulares. Para obtenção do mapa de uso da terra recorreu-se ao processo de classificação de imagens multiespectrais, no qual utilizou-se uma cena obtida a partir do satélite CBERS-2B, cuja resolução espacial é de 20 metros. A imagem foi disponibilizada pelo INPE, sendo datada de 2 de fevereiro de 2010, na órbita 155/ponto 125. O mapa foi gerado no software Spring 5.2, por meio do método supervisionado por pixel utilizando o classificador Maxver. Em relação aos dados tabulares, o modelo trabalha com uma grande quantidade de informações relacionadas ao crescimento das culturas, à ciclagem de nutrientes, bem como parâmetros hidrológicos relacionados ao escoamento e infiltração. Optou-se por utilizar os parâmetros definidos como default pelo modelo, visto que tais atributos são de caracterização complexa e de grande dificuldade de obtenção na literatura nacional.

Após a delimitação das sub-bacias e inserção dos dados pedológicos e de uso da terra, foi realizada a definição das Unidades de Resposta Hidrológica (HRU). Neste trabalho, utilizou-se a opção de múltiplas HRU por sub-bacia, com o intuito de manter um maior detalhamento e evitar a perda ou alteração das informações de entrada, tendo-se ao final a geração de 236 HRUs, distribuídas por um total de 53 sub-bacias.

Os dados climáticos utilizados foram obtidos a partir de seis estações meteorológicas e postos pluviométricos localizados no interior e entorno da área de estudo, cuja localização pode ser observada na Figura 1. As séries climáticas necessárias incluem dados diários de precipitação, temperatura, radiação solar, umidade relativa e velocidade do vento. Foram utilizadas séries temporais compreendendo o período entre 1998 e 2010, representando um total de 13 anos contínuos. Destes 13 anos simulados, dois foram utilizados para aquecimento do modelo, em procedimento conhecido como warmup, visto que, no início da simulação, ocorrem incertezas envolvendo as condições iniciais, principalmente em parâmetros relacionados ao movimento da água no solo.

Para as etapas de calibração e validação dos resultados de vazão, utilizou-se uma série histórica de 11 anos (2000 a 2010) obtida em um posto fluviométrico operado pela COHIDRO, localizado nas proximidades do exutório da bacia (ver Figura 1). Deste total, sete anos foram usados para calibração (2000 a 2006) e quatro anos para a validação (2007 a 2010).

A seleção dos parâmetros a serem utilizados na calibração foi realizada a partir de uma análise de sensibilidade utilizando o método LHS-OAT (MORRIS, 1991), visando avaliar a influência de cada um destes parâmetros na qualidade das respostas do modelo. O processo de calibração foi realizado no software SWAT-CUP utilizando a técnica de otimização Sequential Uncertainty Fitting (SUFI-2), sendo este realizado tanto para vazão mensal quanto para vazão diária. Para avaliar o ajuste entre os dados simulados e os dados observados foram utilizados o Coeficiente de Nash-Sutcliffe Efficiency (NSE), o Coeficiente de Determinação $\left(\mathrm{R}^{2}\right)$ e o Percentual de Tendência (PBIAS).

\section{Simulação do impacto das mudanças climáticas na bacia do Ribeirão do Feijão}

A partir do modelo devidamente calibrado e validado para as condições atuais, torna-se possível realizar o processo de simulação alterando-se os dados de entrada de modo a refletir os diferentes cenários de mudanças climáticas projetados para

Tabela 1 - Projeção do aquecimento médio global da superfície terrestre para o período de 2090 - 2099, em relação à temperatura observada em 1980 - 1999 nos diferentes cenários

\begin{tabular}{|c|c|c|}
\hline Cenários de emissões & $\begin{array}{c}\text { Melhor } \\
\text { estimativa }\left({ }^{\circ} \mathrm{C}\right)\end{array}$ & $\begin{array}{c}\text { Faixa } \\
\text { provável }\left({ }^{\circ} \mathrm{C}\right)\end{array}$ \\
\hline $\begin{array}{l}\text { B1 - Apresenta rápidas mudanças na estrutura econômica mundial, com ênfase em } \\
\text { soluções globais, na sustentabilidade ambiental e na inserção de tecnologias limpas }\end{array}$ & 1,8 & $1,1-2,9$ \\
\hline $\begin{array}{l}\text { A1T - Semelhante ao cenário } \mathrm{A} 1 \mathrm{~B} \text {, porém com uma mudança tecnológica no } \\
\text { sistema energético dando ênfase ao uso intensivo de fontes de energia não fóssil }\end{array}$ & 2,4 & $1,4-3,8$ \\
\hline $\begin{array}{l}\text { B2 - Descreve um mundo com uma população intermediária e ênfase nas soluções } \\
\text { locais para a sustentabilidade econômica, social e ambiental }\end{array}$ & 2,4 & $1,4-3,8$ \\
\hline $\begin{array}{l}\text { A1B - Representa um mundo futuro onde a globalização é dominante, apresentando } \\
\text { crescimento econômico muito rápido, população atingindo o pico em meados do } \\
\text { século e desenvolvimento rápido de tecnologias eficientes, havendo um equilíbrio } \\
\text { entre fontes de energia fósseis e não fósseis }\end{array}$ & 2,8 & $1,7-4,4$ \\
\hline $\begin{array}{l}\text { A2 - Descreve um mundo futuro heterogêneo onde a regionalização é dominante, } \\
\text { com alto crescimento populacional, lento desenvolvimento econômico e mudanças } \\
\text { tecnológicas lentas }\end{array}$ & 3,4 & $2,0-5,4$ \\
\hline $\begin{array}{l}\text { A1FI - Semelhante ao cenário A1B, porém com uma mudança tecnológica no } \\
\text { sistema energético dando ênfase ao uso intensivo de fontes fósseis }\end{array}$ & 4,0 & $2,4-6,4$ \\
\hline
\end{tabular}


as próximas décadas. O Quarto Relatório de Avaliação das Mudanças Climáticas (IPCC, 2007) apresenta uma projeção para o aquecimento médio global da superfície terrestre de acordo com seis diferentes famílias de cenários, obtidos a partir de tendências estimadas de emissão de $\mathrm{CO}_{2}$. $\mathrm{Na}$ Tabela 1 são apresentadas as estimativas e prováveis faixas de incerteza avaliadas para o aquecimento projetado para o final do século XXI, considerando-se como referência as temperaturas observadas entre 1980 e 1999.

Em relação às alterações na precipitação, as projeções realizadas pelo IPCC apontam a ocorrência de verões mais chuvosos e invernos mais secos nas regiões de latitudes médias, especialmente no Sudeste e Sul do Brasil. Para o cenário A1B, por exemplo, estima-se que possa ocorrer um aumento da precipitação entre os meses de dezembro a fevereiro nos últimos anos do século XXI, enquanto nos meses de junho a agosto pode haver uma redução no total precipitado, considerando-se as precipitações atuais como referência.

As projeções de temperatura e precipitação obtidas pelos modelos de clima global (GCMs) são realizadas utilizando um "grid" tridimensional sobre o planeta, tendo resolução global variando entre 250 e $600 \mathrm{~km}$. Desse modo, devido à baixa resolução destes dados, torna-se necessária a realização de procedimentos de downscaling para a aplicação destas projeções em escalas menores.

Neste trabalho, optou-se por utilizar um método de downscaling estatístico baseado no gerador climático estocástico Long Ashton Research Station Weather Generator (LARS-WG), desenvolvido por Semenov e Brooks (1999). O LARS-WG permite gerar séries meteorológicas para localizações específicas através de distribuições de probabilidade semi-empíricas e correlações entre elementos meteorológicos, mantendo-se fiel às estatísticas de uma dada série observada. Para simulação de climas futuros, o gerador é compatível com 15 GCMs utilizados pelo IPCC em suas projeções climáticas, sendo possível realizar simulações tendo como base os cenários de emissões B1, A1B e A2.

Para simulação no LARS-WG foram utilizadas séries históricas de precipitação, temperatura e radiação solar obtidas nas três estações meteorológicas da área de estudo (Figura 1). As séries sintéticas representativas do clima futuro foram geradas aplicando-se transformações tendo como base o modelo de clima global HadCM3, desenvolvido pelo Centro Hadley da Agência Nacional de Meteorologia do Reino Unido. Foram geradas séries sintéticas para três cenários de emissões distintos, sendo eles B1 (baixas emissões), A1B (emissões intermediárias) e A2 (altas emissões), nos períodos de 2050 a 2059 e de 2090 a 2099.

Concomitantemente, a partir dos dados adquiridos nas estações, foi também gerada uma série sintética mantendo-se as características das séries originais, ou seja, sem perturbações simulando as alterações do clima futuro. Tal série foi gerada de modo a servir como cenário de referência ou baseline, visando permitir comparações entre as simulações do clima futuro e o clima atual.

As séries sintéticas geradas foram utilizadas para realimentar o modelo SWAT calibrado para a bacia do Ribeirão do Feijão, de modo a avaliar os impactos das mudanças de precipitação e temperatura na vazão da bacia.

\section{RESULTADOS E DISCUSSÃO}

\section{Calibração e validação do modelo SWAT para a bacia do Ribeirão do Feijão}

Na Tabela 2 são apresentados os parâmetros selecionados para calibração do modelo com base na análise de sensibilidade realizada, a qual permitiu evidenciar aqueles que tiveram maior contribuição nos resultados. Foram selecionados 12 parâmetros, os quais foram ordenados na forma de um ranking conforme sua importância para os resultados de vazão. $\mathrm{O}$ t-stat indica o grau da sensibilidade, em que quanto maior for o seu valor absoluto mais sensível é o parâmetro. Verifica-se que os parâmetros de maior importância estão relacionados com as propriedades do solo (SOL_Z e SOL_AWC) e com o fluxo de água subterrânea (LAT_TTIME).

$\mathrm{Na}$ Tabela 2 são também apresentados os valores ajus-

Tabela 2 - Ranking de parâmetros selecionados para calibração e respectivos valores ajustados

\begin{tabular}{lclcc}
\hline Ranking & Parâmetro & \multicolumn{1}{c}{ Descrição } & t-stat & Valor ajustado \\
\hline 1 & SOL_Z & Profundidade do solo (mm) & $-2,72$ & $-0,36(\mathrm{r})$ \\
2 & LAT_TTIME & Tempo de retorno do fluxo lateral (dias) & $-2,69$ & $1,06(\mathrm{v})$ \\
3 & SOL_AWC & Capacidade de água disponível no solo (mm/mm) & $-2,26$ & $-0,39(\mathrm{r})$ \\
4 & EPCO & Fator de compensação de retirada de água do solo pelas & $-1,96$ & $0,27(\mathrm{v})$ \\
5 & plantas & $-1,51$ & $30,7(\mathrm{v})$ \\
6 & GW_DELAY & Intervalo de tempo para recarga do aquífero (dias) & $-1,28$ & $-0,54(\mathrm{r})$ \\
7 & CN2 & Curva-Número inicial para umidade II & $-1,16$ & $91,9(\mathrm{v})$ \\
8 & CH_K2 & Condutividade hidráulica efetiva do canal $\left(\mathrm{mm} \cdot \mathrm{h}^{-1}\right)$ & $-1,08$ & $0,16(\mathrm{r})$ \\
9 & SOL_BD & Densidade do solo (g/cm $\left.{ }^{3}\right)$ & 1,01 & $0,17(\mathrm{v})$ \\
10 & ALPHA_BNK & Fator de escoamento de base (dias) & $-0,73$ & $13,2(\mathrm{v})$ \\
11 & SURLAG & Coeficiente de retardo do escoamento superficial & $-0,31$ & $0,38(\mathrm{v})$ \\
12 & CH_N2 & Número de Manning & $-0,23$ & $-0,4(\mathrm{r})$ \\
\hline
\end{tabular}

(r): Ajuste por meio de multiplicação do valor original pelo valor indicado. (v): Ajuste por meio da substituição do valor original pelo valor indicado 
tados para cada um dos parâmetros após as sucessivas iterações através do método de otimização SUFI-2. Os parâmetros SOL_Z, SOL_AWC, CN2, SOL_BD e SOL_K, por refletirem características dos tipos de solo e usos da terra, foram modificados através do método de multiplicação de valores, sendo alterados de forma distribuída, embora sempre na mesma proporção. Já os demais parâmetros, por serem únicos e não variarem espacialmente na bacia, foram modificados por meio da substituição de valores. Os procedimentos de calibração foram feitos primeiramente para a simulação mensal e depois, após o devido ajuste fino, foram aplicados à simulação diária.

Na Figura 2 é apresentado o hidrograma da simulação de vazão mensal, onde é possível visualizar o ajuste dos dados simulados em relação à série observada no posto fluviométrico. Os métodos estatísticos utilizados para avaliar o desempenho do modelo são apresentados na Tabela 3.

Para a simulação mensal foram obtidos valores de NSE de 0,73 na calibração e 0,78 na validação, permitindo-se classificar o desempenho do modelo como 'bom' e 'muito bom' segundo Moriasi et al. (2007). O coeficiente NSE é largamente utilizado em estudos hidrológicos por ser altamente influenciado por valores extremos dentro das séries analisadas, sendo, portanto, bastante sensível aos eventos de cheia. Tal fato explica o melhor desempenho do modelo para o período de validação, visto a menor ocorrência de picos extremos de vazão quando comparado ao período de calibração.
Através da análise visual da simulação mensal, verifica-se ainda que, no geral, os dados simulados foram levemente superiores aos dados observados em grande parte do período. Tal fato é refletido pelo índice PBIAS, que diz respeito ao percentual de viés entre as séries observada e simulada, o qual foi de 5,5\% na calibração e $8,9 \%$ na validação. Desse modo, no período calibrado as vazões foram superestimadas em $5,5 \%$, enquanto na validação a superestimação foi de $8,9 \%$. O período de calibração apresentou menor PBIAS devido a espaços de tempo onde a vazão foi subestimada, como, por exemplo, nas estações secas dos anos de 2000 e 2001, conforme pode ser observado pelo hidrograma. Segundo Moriasi et al. (2007), os valores de PBIAS para ambos os períodos são considerados muito bons.

Na Figura 3 é apresentado o hidrograma para a simulação diária. Os valores de NSE obtidos foram de 0,57 para a calibração e 0,63 para a validação, sendo ambos considerados como 'satisfatórios'. Em ambos os períodos o desempenho diário foi inferior ao desempenho mensal, o que é bastante esperado visto a maior dificuldade em simular eventos extremos em menor escala de tempo, especialmente em bacias onde o tempo de concentração é inferior a um dia. Tal fato é refletido no hidrograma, conforme pode-se observar a maior disparidade entre os valores simulados e observados em alguns picos de cheia, resultando, portanto, em menores valores de NSE quando comparados às simulações mensais.

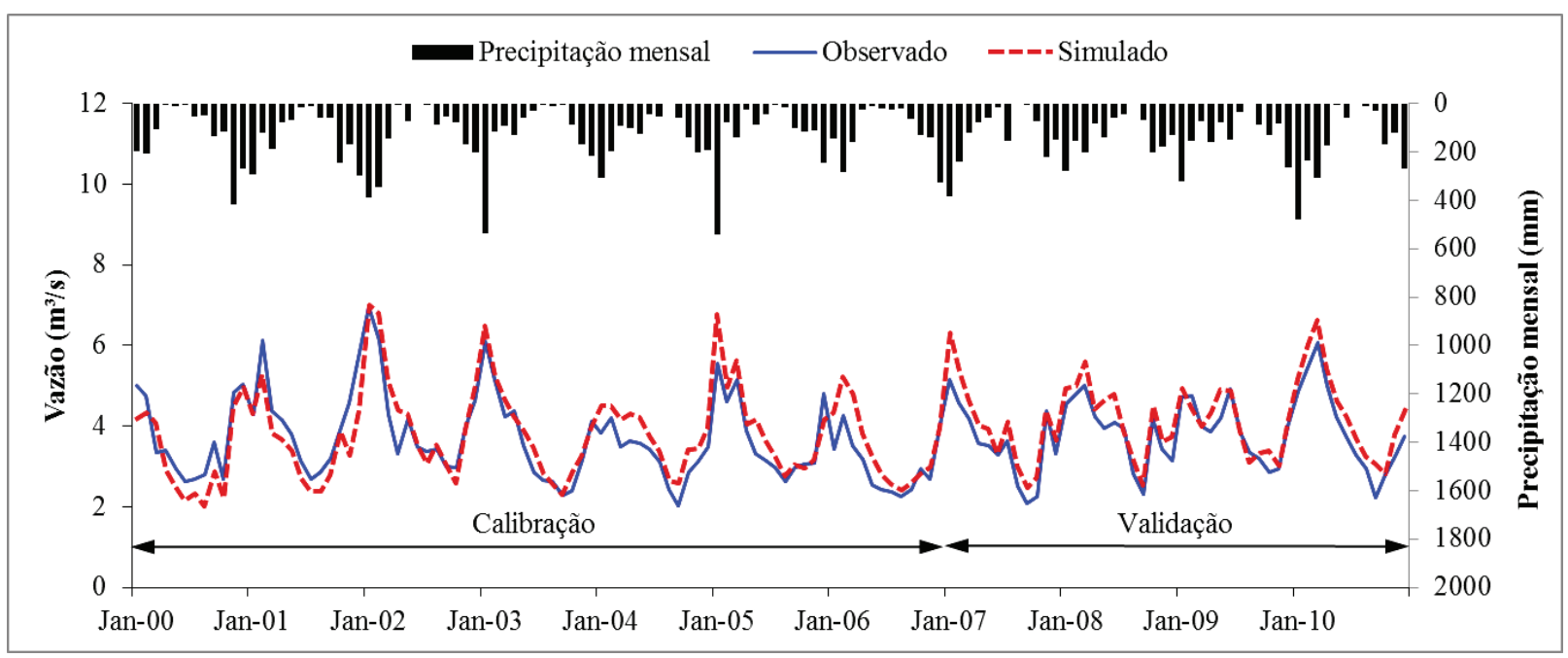

Figura 2 - Simulação de vazões mensais nos períodos de calibração e validação

Tabela 3 - Estatísticas de verificação do ajuste do modelo para vazão mensal e diária nas etapas de calibração/validação e classificação dos resultados de acordo com Moriasi et al. (2007)

\begin{tabular}{|c|c|c|c|c|c|c|}
\hline \multirow{2}{*}{ Simulação } & \multicolumn{3}{|c|}{ Calibração } & \multicolumn{3}{|c|}{ Validação } \\
\hline & NSE & PBIAS & $\mathbf{R}^{2}$ & NSE & PBIAS & $\mathbf{R}^{2}$ \\
\hline Mensal & $\begin{array}{l}0,73 \\
\text { (Bom) }\end{array}$ & $\begin{array}{c}5,5 \% \\
\text { (Muito Bom) }\end{array}$ & 0,76 & $\begin{array}{c}0,78 \\
\text { (Muito Bom) }\end{array}$ & $\begin{array}{c}8,9 \% \\
\text { (Muito Bom) }\end{array}$ & 0,91 \\
\hline Diária & $\begin{array}{c}0,57 \\
\text { (Satisfatório) }\end{array}$ & $\begin{array}{c}-6,6 \% \\
\text { (Muito Bom) }\end{array}$ & 0,57 & $\begin{array}{c}0,63 \\
\text { (Satisfatório) }\end{array}$ & $\begin{array}{l}10,7 \% \\
\text { (Bom) }\end{array}$ & 0,75 \\
\hline
\end{tabular}




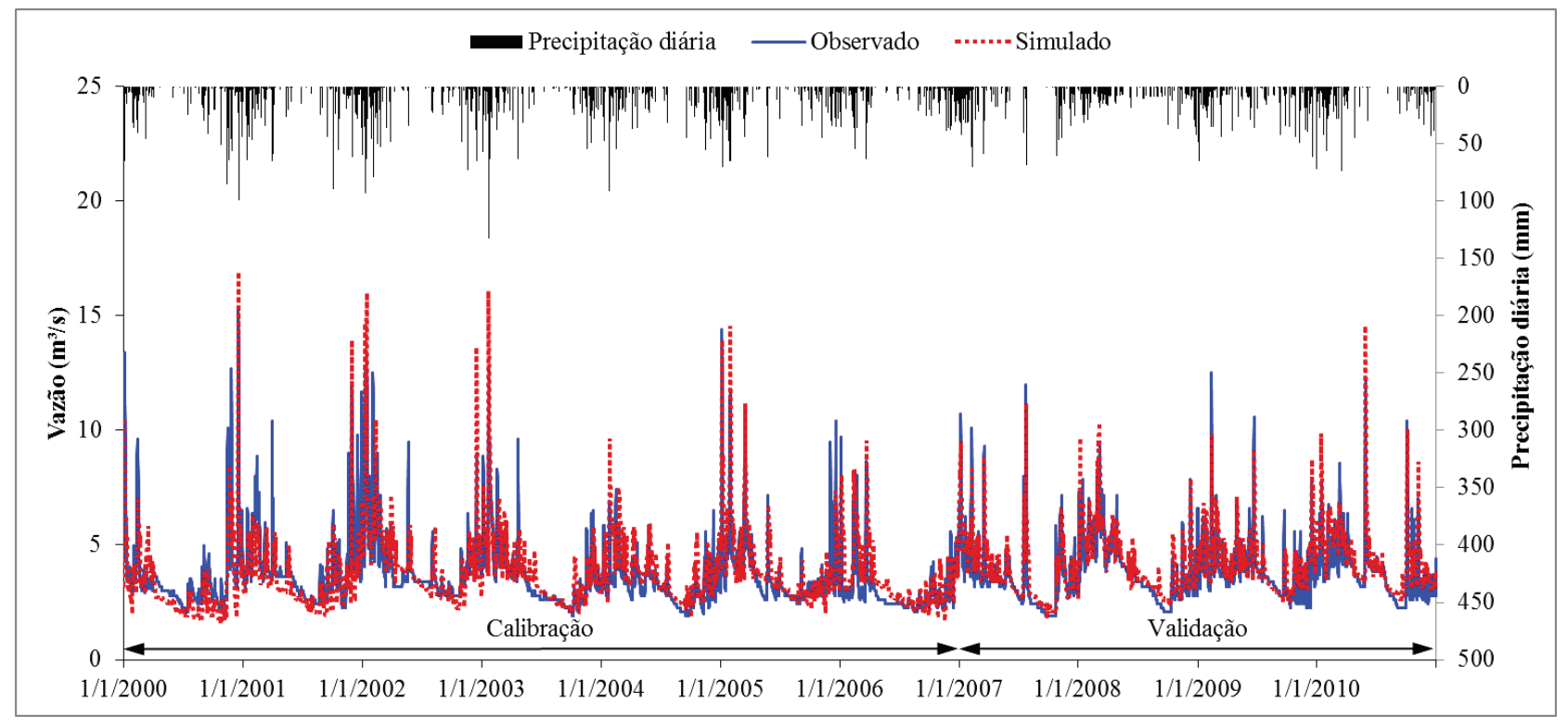

Figura 3 - Simulação de vazões diárias nos períodos de calibração e validação

Em relação ao PBIAS, o período de calibração apresentou uma subestimativa de vazões de $-6,6 \%$, o qual pode ser observado tanto nos períodos secos de alguns anos como em alguns eventos de cheia subestimados. Já o período de validação apresentou PBIAS de 10,7\%, indicando superestimação de vazões, a qual pode ser facilmente observada pelo hidrograma no período compreendido entre o final de 2009 e início de 2010 , onde os valores simulados foram notadamente superiores aos observados. Para a calibração o desempenho do índice PBIAS foi considerado 'muito bom', enquanto para a validação foi considerado 'bom'.

Apesar do desempenho inferior observado nas simulações sob passo de tempo diário, considera-se que o modelo SWAT apresentou uma boa adequação para sua utilização na bacia, obtendo-se estimativas de boa precisão e sem tendências enviesadas, tornando-o apto para realizar simulações sob diferentes cenários de mudança climática.

\section{Simulação dos impactos das mudanças climáticas na vazão}

Na Figura 4 são apresentados os acréscimos na temperatura média mensal visando simular as condições climáticas nas décadas de 2050 e 2090, enquanto a Figura 5 apresenta os percentuais de variação da precipitação para estes mesmos períodos. Tais estimativas foram geradas no software LARS-WG a partir do modelo de clima global HadCM3, tendo como base os cenários de emissões B1 (baixas emissões), A1B (cenário moderado) e A2 (altas emissões).

Em relação à temperatura, verifica-se que em todos os cenários os maiores acréscimos ocorreram nos meses de julho, agosto e setembro, chegando a quase $6^{\circ} \mathrm{C}$ para a década de 2090 no cenário A2. Para a década de 2050, as alterações variaram entre $1^{\circ} \mathrm{C}$ e $3^{\circ} \mathrm{C}$, sendo os menores valores observados no cenário $\mathrm{B} 1$ e os maiores no $\mathrm{A} 2$.

Com relação às mudanças na precipitação, verificou- se um acréscimo nos totais de chuva mensal para os meses de outubro a janeiro em todos os cenários. Nos demais meses, alguns cenários apontaram incremento na quantidade de chuvas enquanto outros mostraram uma redução. Para o mês de agosto apenas o cenário 2050-B1 apresentou acréscimo, enquanto nos outros cenários houve redução, sendo esta de 33\% no cenário 2090 - A1B. Outros cenários que apontaram alta redução das chuvas nos meses mais secos foram 2050 - A2 e 2090 - B1.

Estima-se que tais modificações na temperatura e na precipitação seriam suficientes para ocasionar diversos impactos no nível de bacia hidrográfica, como alterações no crescimento das culturas, na produtividade agrícola, na frequência e intensidade de cheias e na disponibilidade hídrica. Desse modo, visando-se obter estimativas do impacto das mudanças climáticas na vazão do Ribeirão do Feijão, tais padrões de mudança do clima foram aplicados às séries diárias obtidas nas estações da bacia, de modo a obter séries sintéticas representativas do clima nas décadas de 2050 e 2090. Estas séries sintéticas foram utilizadas como dado de entrada no modelo SWAT já calibrado para a bacia.

Nas Figuras 6 e 7 são apresentadas as variações percentuais nos padrões de vazões mensais para os cenários considerados, tendo como referência os resultados de uma simulação realizada utilizando-se uma série climática não perturbada (baseline) para servir de comparação. Apesar de o modelo hidrológico ter sido calibrado para o passo de tempo diário, os resultados das simulações foram resumidos por meio de médias mensais, de modo a permitir a análise sazonal das vazões e a comparação com os padrões atuais.

Através da análise da Figura 6, referente às simulações para a década de 2050, verifica-se que nos cenários B1 e A1B houve um aumento nas médias mensais de vazão em todos os meses, sendo este mais notável nos meses de outubro, novembro e dezembro, chegando pouco acima de $10 \%$. Para o cenário B1 o acréscimo de vazão situou-se entre 2 e 10,7\% enquanto para o cenário A1B o aumento foi entre 4,8 e 10,4\%. Já para o cenário de altas emissões A2, verifica-se uma diminuição da 


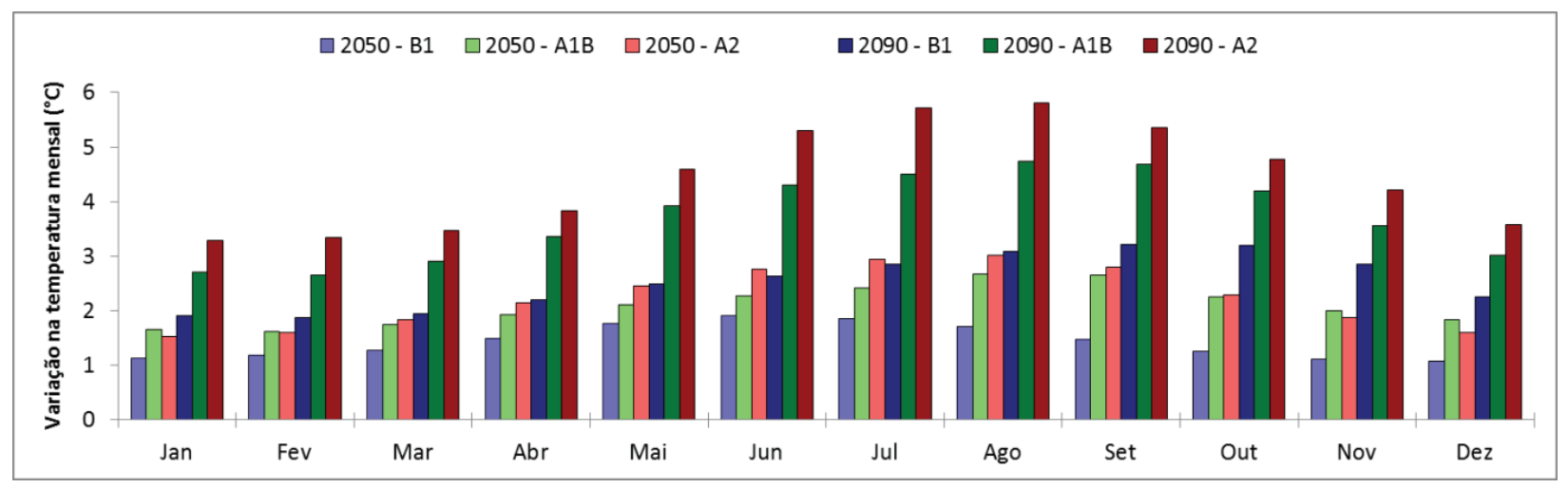

Figura 4 - Acréscimos na temperatura média mensal para os cenários simulados nas décadas de 2050 e 2090 , tendo como referência os padrões de temperatura atuais

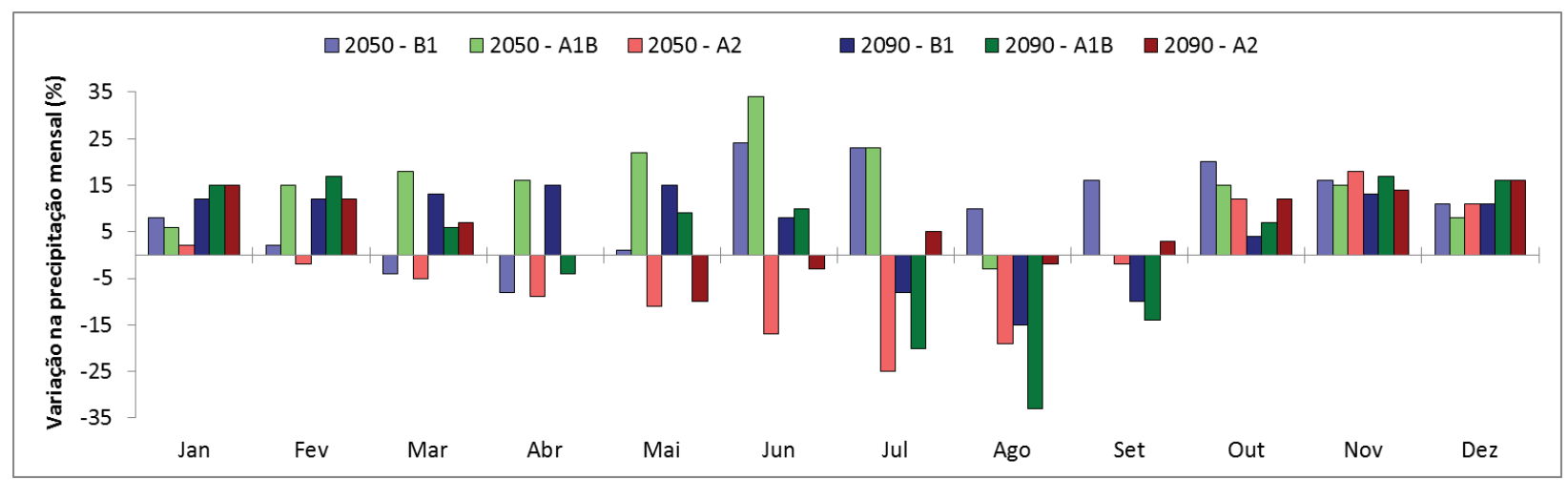

Figura 5 - Alterações percentuais na precipitação média mensal para os cenários simulados nas décadas de 2050 e 2090 , tendo como referência os padrões de precipitação atuais

vazão mensal no período de maio a setembro, variação esta compreendida na faixa de $-1,5 \%$ a $-3 \%$. Na estação chuvosa (novembro a março), a vazão apresentou acréscimos, tendo máximo em dezembro $(7,9 \%)$.

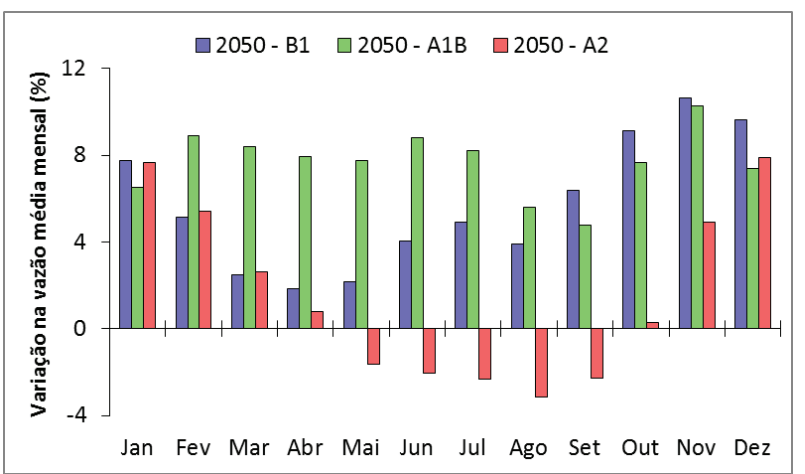

Figura 6 - Variação na vazão média mensal na década de 2050 para os cenários considerados

Tais resultados para a década de 2050 estão fortemente ligados às modificações nos padrões de chuva e temperatura apresentados nas Figuras 4 e 5. Para os três cenários a temperatura média sofreu um acréscimo de 1 a $3^{\circ} \mathrm{C}$, enquanto para a precipitação houve aumento em praticamente todos os meses para os cenários B1 e A1B. No cenário A2 (altas emissões), a redução da precipitação no período seco associado ao aumento das temperaturas acabou por gerar os decréscimos de vazão observados entre maio e setembro.

Na Figura 7 são apresentadas as variações de vazão mensal para a década de 2090, onde se verifica que nos três cenários houve meses onde a variação de vazão mensal foi negativa.

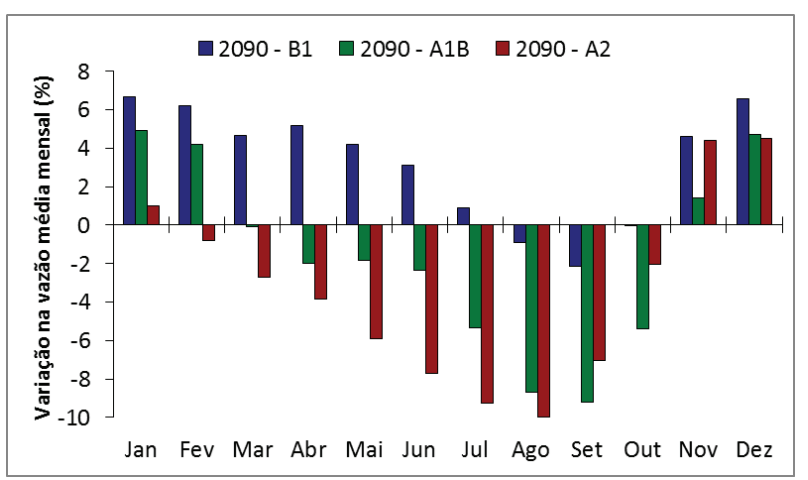

Figura 7 - Variação na vazão média mensal na década de 2090 para os cenários considerados

No cenário de menores emissões B1 ocorreram vazões negativas apenas em agosto $(-0,9 \%)$ e setembro $(-2,1 \%)$, enquanto nos demais meses a variação foi positiva, tendo seu máximo em janeiro (6,7\%). Para o cenário A1B, de emissões intermediárias,

ocorreram decréscimos entre os meses de março a outubro, 
tendo a variação máxima de $-9,2 \%$ em setembro, enquanto os demais meses apresentaram acréscimos. As menores vazões foram observadas no cenário de altas emissões A2, o qual apresentou redução em nove meses consecutivos (fevereiro a outubro), com máxima variação de $-10,1 \%$ no mês de agosto.

Ainda com relação a estes cenários, verifica-se que mesmo com maiores reduções de precipitação no cenário A1B, conforme se pode observar na Figura 5, o cenário A2 apresentou as menores vazões. Tal fato pode ser atribuído ao maior aumento da temperatura média mensal no cenário $\mathrm{A} 2$, denotando a alta sensibilidade das rotinas de modelagem do SWAT às alterações na temperatura.

Tendo em vista as estimativas mensais obtidas para o final do século, verifica-se que o pior caso em relação à disponibilidade hídrica ocorreu no cenário $\mathrm{A} 2$, seguido do cenário A1B, onde mais da metade do ano apresentou redução da vazão. Uma redução dessa ordem poderia ocasionar um agravamento dos conflitos relacionados ao uso da água na bacia, visto que, além de ser utilizada como manancial de abastecimento público, há uma grande demanda de água destinada à irrigação dos cultivos agrícolas da bacia, em especial da cana-de-açúcar, a qual tem apresentado um acelerado ritmo de expansão. Por outro lado, nos meses chuvosos o aumento da vazão pode ocasionar problemas relacionados a inundações, principalmente nas áreas impermeabilizadas da bacia, além de alterações na qualidade da água devido aos eventos de chuva extremos.

\section{CONCLUSÕES}

O modelo SWAT foi aplicado e calibrado para a bacia do Ribeirão do Feijão tanto em passo diário como mensal. Os resultados indicaram que o modelo conseguiu prever com uma boa faixa de precisão as vazões ocorrentes na bacia, mostrando-se, portanto, apto à realização de simulações sob diferentes cenários de mudanças climáticas.

As simulações realizadas permitiram constatar a alta sensibilidade do modelo em relação às perturbações realizadas nas séries climáticas originais, permitindo-se quantificar numericamente os possíveis impactos das mudanças climáticas na vazão da bacia do Ribeirão do Feijão. Constatou-se que as simulações para a década de 2050 apresentaram uma tendência de aumento da vazão mensal nos cenários simulados devido ao aumento das precipitações. Já nas simulações para o final do século, devido à redução na precipitação, houve decréscimos significativas nos padrões mensais de vazão durante os meses mais secos, podendo tal diminuição chegar a até $10 \%$. Por outro lado, nos meses de novembro, dezembro e janeiro as simulações apontaram aumento na vazão em todos os cenários para a década de 2090.

Tais predições da resposta hidrológica da bacia às mudanças projetadas no clima futuro podem fornecer subsídios para elaboração de novas estratégias de gestão dos recursos hídricos, permitindo se antecipar possíveis problemas de disponibilidade hídrica no futuro, bem como auxiliar na elaboração de políticas de mitigação de impactos decorrentes da mudança do clima.

\section{REFERÊNCIAS}

ARNOLD, J. G. et al. Soil and water assessment tool: input/ output file documentation. Texas: Soil And Water Research Laboratory \& Blackland Research Center, 2009. 662 p.

ARROIO JUNIOR, P. P. Avaliação da produção e transporte de sedimentos na bacia hidrográfica do Rio Itaqueri, municípios de Itirapina e Brotas - SP. 2013. 112 f. Dissertação (Mestrado em Ciências da Engenharia Ambiental) - Universidade de São Paulo, São Carlos, 2013.

FICKLIN, D. L. et al. Climate change sensitivity assessment of a highly agricultural watershed using SWAT. Journal of Hydrology, v. 374, p. 16-29, 2009.

INTERGOVERNMENTAL PANEL ON CLIMATE CHANGE - IPCC. Climate Change 2013: The Physical Science Basis. Contribution of Working Group I to the Fifth Assessment Report. Cambridge, UK: Cambridge University Press, 2013. 1536 p.

INTERGOVERNMENTAL PANEL ON CLIMATE CHANGE - IPCC. Climate Change 2007: The Physical Science Basis. Contribution of Working Group I to the Fourth Assessment Report. Cambridge: Cambridge University Press, 2007. 996 p.

LELIS, T. A.et al. Impactos causados pelas mudanças climáticas nos processos erosivos de uma bacia hidrográfica: Simulação de cenários. Revista Ambiente \& Água, v. 6, p. 282-294, 2011.

MARENGO, J. A. Água e mudanças climáticas. Estudos Avançados, São Paulo, v. 22, n. 63, p. 83-96, 2008.

MELLO, E. L. et al. Efeito das mudanças climáticas na disponibilidade hídrica da bacia hidrográfica do Rio Paracatu. Eng. Agrícola, Jaboticabal, v. 28, p.635-644, 2008.

MORIASI D. N. Model evaluation guidelines for systematic quantification of accuracy in watershed simulations. Transactions of the American Society of Agricultural and Biological Engineers, v. 50, n. 3, p. 885-900, 2007.

MORRIS, M. D. Factorial sampling plans for preliminary computational experiments. Technometrics, v. 33, n.2, p. 161 174, 1991.

OLIVEIRA, J. B.; PRADO, H. Levantamento pedológico semidetalhado do Estado de São Paulo: Quadrícula de São Carlos. Memorial descritivo. Campinas: IAC, 1984. 110 p.

PERAZZOLI, M.; PINHEIRO, A.; KAUFMANN, V. Assessing the impact of climate change scenarios on water resources in southern Brazil. Hydrological Sciences Journal, v. 58, n. 1, p. 77-87, 2013. 
SEMENOV, M. A.; BROOKS, R. J. Spatial interpolation of the LARS-WG weather generator in Great Britain. Climate Research, v. 11; p. 137-148, 1999.

SRINIVASAN, R.; ARNOLD, J. G. Integration of a basin-scale water quality model with GIS. Water Research, v. 30, p. 453-462, 1994.

TIM, U. S. Emerging technologies for hydrologic and water quality modeling research. Transactionsofthe $A S A E$, v. 39, n. 20, p. 465-476, 1996.

TUCCI, C. E. M. Mudanças climáticas e impactos sobre os recursos hídricos no Brasil. Ciência \& Ambiente, v. 34, p. 137$156,2007$.

VALERIO, E. L. S. Avaliação dos efeitos de alterações na precipitação devido a mudanças climáticas no regime hidrológico da bacia do Rio Paraguaçu, BA. 2014. 123 f. Dissertação (Mestrado em Recursos Hídricos e Saneamento) - Universidade Federal de Alagoas, Maceió, 2014.

WAGESHO, N.; JAIN, M. K.; GOEL, N. K. Effect of Climate Change on Runoff Generation: Application to Rift Valley Lakes Basin of Ethiopia. Journal of Hydrologic Engineering, v. 18, p. 1048-1063, 2013.

ZAHABIYOUN, B. et al. Assessment of Climate Change Impact on the Gharesou River Basin Using SWAT Model. Clean - Soil, Air, Water, v. 41, n. 6, p. 601-609, 2013. 